\title{
A new quantification method for mechanical dyssynchrony with three-dimensional echocardiography; segmental time and volume loss for prediction of response to cardiac resynchronisation therapy
}

\author{
J. A. van der Heide $\cdot$ M. F. A. Aly $\cdot$ S. A. Kleijn • \\ J. van Dijk • O. Kamp
}

Received: 8 May 2011 / Accepted: 17 January 2012/Published online: 3 February 2012

(C) The Author(s) 2012. This article is published with open access at Springerlink.com

\begin{abstract}
A novel method to assess left ventricular (LV) mechanical dyssynchrony using three-dimensional echocardiography (3DE) and semi-automated border detection was investigated, which might be superior in prediction of response to cardiac resynchronisation therapy (CRT) compared to traditional measures that rely solely on segmental time-to-contraction. Twentyeight heart failure patients underwent real-time 3DE before CRT and at 6-12 months follow-up. Analysis of 3DE was performed using TomTec Research-Arena software featuring semi-automated endocardial border detection. The following echocardiographic parameters were calculated in a 16-segment model: areas under segmental time-volume-curves (STV); delay between contraction of the earliest and latest segment
\end{abstract}

J. A. van der Heide - M. F. A. Aly .

S. A. Kleijn · J. van Dijk · O. Kamp ( $\square)$

Department of Cardiology, Institute for Cardiovascular

Research (IcaR-VU), VU Medical Center, VU University

Medical Center, De Boelelaan 1117, 1081 HV

Amsterdam, The Netherlands

e-mail: O.Kamp@vumc.nl

J. A. van der Heide - M. F. A. Aly .

S. A. Kleijn · J. van Dijk · O. Kamp

Department of Cardiology, Institute for Cardiovascular

Research (IcaR-VU), VU Medical Center, VU University

Medical Center, P.O. Box 7057, 1007 MB Amsterdam,

The Netherlands
(L-E); and standard deviation of segmental time-tocontraction (SDI). Response to CRT was defined as $\geq 10 \%$ decrease in LV end-systolic volume at followup. Baseline Pre-STV had a higher sensitivity than SDI for prediction of response (94 vs 67\%, respectively), with equal specificity (78\%) and a higher area under receiver operator characteristic curve. In contrast, L-E had a sensitivity of $83 \%$ and a specificity of $56 \%$. Using $3 \mathrm{DE}$, methods that combine segmental time-tocontraction with segmental contractility might improve LV dyssynchrony assessment compared to traditional methods based on segmental time-to-contraction alone. Pre-STV might be a better predictor of response to CRT than SDI.

Keywords Cardiac resynchronisation therapy ·

Three-dimensional echocardiography · Left ventricle . Dyssynchrony

$\begin{array}{ll}\text { Abbreviations } \\ \text { 3DE } & \text { Three-dimensional echocardiography } \\ \text { CRT } & \text { Cardiac resynchronsation therapy } \\ \text { EDV } & \text { End-diastolic volume } \\ \text { ESV } & \text { End-systolic volume } \\ \text { EF } & \text { Ejection fraction } \\ \text { L-E } & \text { Latest minus earliest segment } \\ \text { LV } & \text { Left ventricle } \\ \text { Pre-STV } & \text { Pre-systolic time-volume loss } \\ \text { Post-STV } & \text { Post-systolic time-volume loss } \\ \text { SDI } & \text { Systolic dyssynchrony index } \\ \text { TDI } & \text { Tissue Doppler Imaging }\end{array}$




\section{Introduction}

Heart failure remains a complex clinical syndrome. While optimal medical therapy improves survival, cardiac resynchronisation therapy (CRT) has been proven as an effective adjuvant treatment for patients with moderate-to-severe systolic heart failure and wide QRS on optimal medical therapy [1]. However, despite these impressive results, approximately onethird of patients fail to improve after CRT [2]. While the precise mechanisms responsible for the benefits from CRT are not fully understood, the reduction of ventricular dyssynchrony following CRT has been shown to produce favourable acute haemodynamic and neurohormonal changes as well as ventricular remodelling with concomitant symptomatic improvement [2]. Consequently, different imaging-based methods for the assessment of myocardial dyssynchrony have been intensively investigated to improve the prediction of response to CRT [3]. The ideal parameter for the selection of patients for CRT should be simple and practical, and it should not require an elaborate off-line analysis with low reproducibility [4].

Most echocardiographic methods to assess left ventricular (LV) mechanical dyssynchrony rely on timing differences of contraction of individual LV segments, for example Tissue Doppler Imaging (TDI) $[5,6]$ and three-dimensional echocardiography (3DE) [3, 7-9]. Although poor correlation between 3DE and TDI has been observed [10-12], these methods have been found able to predict response to CRT in singlecenter studies $[6,10,11]$. By contrast, however, the multi-center PROSPECT-study [13] thoroughly evaluated a wide range of echocardiographic parameters in response to CRT, with disappointing results. Possibly, low reproducibility of various parameters reported in this study might have caused their low predictive values. Time difference between lateral and septal peak systolic wall velocity measured by TDI Doppler achieved an area under receiver operator characteristic (ROC) curve of only 0.61 for the ability to predict LV end-systolic volume (ESV) reduction; reproducibility of this parameter was not reported. The abovementioned methods are based on measurement of timing of contraction of individual LV segments, but it appears that additional factors should be assessed in prediction of response to
CRT. We feel that hitherto, segmental differences in contractility (as opposed to segmental differences in timing) have been consistently overlooked.

Assessment of LV mechanical dyssynchrony by $3 \mathrm{DE}$ is based on semi-automatic endocardial border detection [14-16]. These systems use segmental timevolume curves which give detailed regional information on LV function. When using software featuring segmental time-volume curves, we observed two distinct patterns: segments with a long contraction delay with preserved (albeit delayed) endocardial inward motion, and segments with a similarly long contraction delay but with decreased endocardial inward motion. When these latter segments are resynchronized by CRT, LV function might fail to improve because irrespective whether those severely hypokinetic segments contract synchronously or not, they contribute very little to global LV function and resynchronisation may not cause significant improvement.

In the present study, we investigate the novel measure of integrating area under time-volume curves for assessment of LV mechanical dyssynchrony in heart failure patients. We hypothesize that this approach, in comparison to traditional 3DE parameters of LV mechanical dyssynchrony, may be of additional value in prediction of response to CRT.

\section{Methods}

A total of 28 heart failure patients were included, with a good echocardiographic apical window and who were eligible for CRT by traditional criteria (ejection fraction $[\mathrm{EF}] \leq 35 \%$, NYHA class III-IV despite optimal medical therapy and a QRS complex $\geq 120 \mathrm{~ms}$. Exclusion criteria were atrial fibrillation, poor echocardiographic image quality and more than moderate mitral regurgitation. Before permanent pacemaker implantation, NYHA class was assessed, 6-min walk test was performed and a Minnesota living with heart failure questionnaire score was obtained. Furthermore, patients underwent real-time 3DE. These measurements were repeated at 6-12 months follow-up. Off-line, 3DE analysis was performed. Predictive values of response to CRT of baseline echocardiographic parameters were calculated as described below. 
Real-time 3DE

For 3DE, a Philips iE 33 ultrasound machine in combination with an X-4 matrix array transducer was used, according to a method described earlier [15]. Patients underwent 3DE in left lateral decubitus position. Recordings were made from the apical window and care was taken to encompass the entire LV in the data set. Gain and compress settings were adjusted to yield optimal endocardial border definition and depth was adjusted to include aortic and mitral valves at the highest possible frame rate. During a short breath-hold, a full volume consisting of 4 partial volumes was acquired. When during acquisition an extrasystole occurred or a motion artifact was observed, the acquisition was discarded. Acquisitions were stored on CD-ROM for later analysis.

Image analysis

Off-line, TomTec Research-Arena 3.0 software featuring semi-automated endocardial border detection was used. The software loads an echo acquisition and shows a 2-chamber, 3-chamber, and 4-chamber view on screen, which can be manually aligned with the LV central axis if necessary. The operator traces endocardial borders in all three views, in end-diastole and endsystole. The software then automatically detects the endocardial borders in 3 dimensions, throughout the cardiac cycle, and divides the LV in a 16-segment model. It calculates LV volumes and EF as well as systolic dyssynchrony index (SDI), defined as the standard deviation of times from QRS complex tot minimal segmental volume; and latest minus earliest segment (L-E), which is defined as the time between earliest and latest segment to reach minimal volume. Both SDI and L-E are expressed as a percentage of RR-interval.

\section{Pre- and Post-STV}

Furthermore, the software calculates Pre-systolic time-volume loss (Pre-STV), which is defined by the area under time-volume curve, as shown in Fig. 1. In calculation of Pre-STV, global end-systolic time is defined as the moment of minimal LV volume. However, the moment of minimal LV volume is the moment that the sum of all segmental volumes is at its minimum, which does not necessarily mean that at that

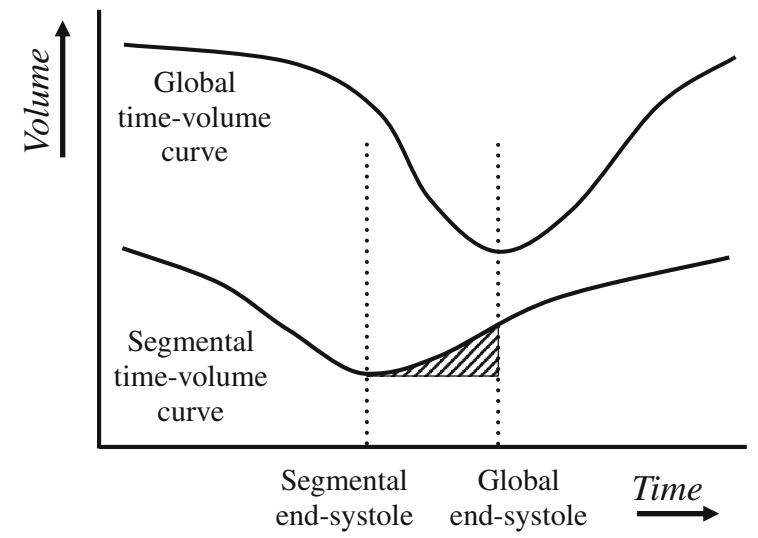

Fig. 1 The time-volume loss concept. A global and a segmental time-volume curve are shown. Clearly, the segment shows early contraction. Segmental end-systolic time and volume are measured. Furthermore, time between global and segmental end-systole are measured, and segmental volume difference between global and segmental end-systole. Pre-STV is defined as area under time-volume curve as indicated by the barred area. Thus, a segment with a large time difference but with low contractility will be assigned a low value. Only segments with both large time differences and high contractility, that are expected to yield the most improvement in CRT, will be assigned high values. Pre-STV is the sum of all segments that reach minimal segmental volume before global end-systole

moment, each individual segmental volume is at its minimum. On the contrary; as is evident from the concept of SDI, each segment reaches its individual minimal volume at different times, which are further spread apart as dyssynchrony worsens. In measurement of Pre-STV, not only timing of minimal volume, but also segmental volume difference between the moments of global and individual minimal volumes is assessed. An individual segment that has reached minimal volume shortly before global end-systole, will be in an early phase of relaxation at global endsystole, with a slight increase in segmental volume. Pre-STV is not only dependent of timing difference but also of this volume difference; the area-undercurve, indicated in Fig. 1, is measured. Pre-STV is the sum of values of all segments that reach minimal segmental volume before global end-systole. Because the $\mathrm{X}$-axis of a time-volume curve is expressed in percentage of RR-interval and the Y-axis is expressed in $\mathrm{ml}$, Pre-STV is expressed in $\mathrm{ml} \times$ percentage of RR-interval.

The example given in Fig. 1 shows a segment with early segmental minimal volume, but there is of course also the possibility of late segmental minimal volume. 
These segments are calculated in a similar fashion; the value is called Post-STV. Finally, (total) STV is defined as the sum of Pre- and Post-STV.

The rationale of Pre- and Post-STV is that they do not only assess time to contraction but also segmental LV function. This way, values of segments that show dyssynchrony in combination with decreased contractility are low, which might be of additional value in prediction of response to CRT. Pre- and Post-STV might serve as a correction of SDI for regional contractility.

Follow-up

After 6-12 months, patients returned for repeat 3DE, all parameters mentioned above were measured and compared to their values at baseline. Response was defined as a $\geq 10 \%$ reduction in ESV compared to baseline and predictive value for response of the abovementioned parameters was calculated [7, 17].

Reproducibility

For assessment of reproducibility, a subgroup of 15 patients was analyzed by two independent observers. The first observer then repeated analysis after 3 weeks.

\section{Statistics}

Values were expressed as average \pm standard deviation, in $\mathrm{ml}$ and $\mathrm{ms}$ or percentage of the $\mathrm{R}-\mathrm{R}$ interval. Comparison of values at baseline and follow-up of responders and non-responders was performed using the student's T-test. Predictive value of response was calculated by ROC curve calculation and by sensitivity, specificity, negative (NPV) and positive predictive value (PPV) calculation. A $p$-value $<0.05$ was considered statistically significant. Reproducibility was assessed using Bland-Altman analysis.

\section{Results}

For baseline patient characteristics, see Table 1 . There was a majority of males and a high percentage of patients with ischemic heart disease in our patient group. Beta-blocker use was relatively low.
Table 1 Baseline clinical patient characteristics

\begin{tabular}{ll}
\hline Age & $65 \pm 10$ years \\
Males/females & $21 / 7$ \\
Ischemic heart disease & $63 \%$ \\
ACEI/ARB use & $79 \%$ \\
Beta-blocker use & $61 \%$ \\
Diuretic use & $86 \%$ \\
Potassium-sparing diuretic use & $54 \%$ \\
\hline
\end{tabular}

Echocardiographic and clinical parameters at baseline and at follow-up are given in Table 2. All patients underwent CRT and follow-up was $100 \%$. There were 8 non-responders and 20 responders in this patient cohort.

Responders demonstrated a significant decrease in LV volumes (by definition in ESV) and increase in EF whereas non-responders demonstrated a non-significant increase in LV volumes and no change in EF.

L-E at baseline was significantly higher in responders. At follow-up, it showed a significant decrease in responders and no change in non-responders. SDI showed a similar pattern. There was a large and significant difference in Pre-STV at baseline between responders and non-responders. At follow-up, PreSTV decreased significantly in responders with no change in non-responders. Post-STV at baseline was not significantly different in non-responders and in responders. No significant changes were observed during follow-up. There was a large and significant difference between Pre- and Post-STV at baseline $(p<0.01)$.

Of the clinical parameters, the decrease in BNP (brain natriuretic peptide) at follow-up reached borderline statistical significance, and there was no significant difference between baseline and followup. NYHA class and QOL (Minnesota Living with Heart Failure questionnaire) score improved equally in responders and non-responders whereas 6-MWT (six-min walk test) increased significantly only in responders.

Predictive values for response to CRT of selected echocardiographic parameters are given in Table 3. Pre-STV had the highest sensitivity and specificity and NPV and PPV of all parameters except for SDI, which had equal specificity. Area under ROC curve of PreSTV was not significantly higher than that of SDI $(p=0.38)$. L-E, SDI and Pre-STV showed significant 


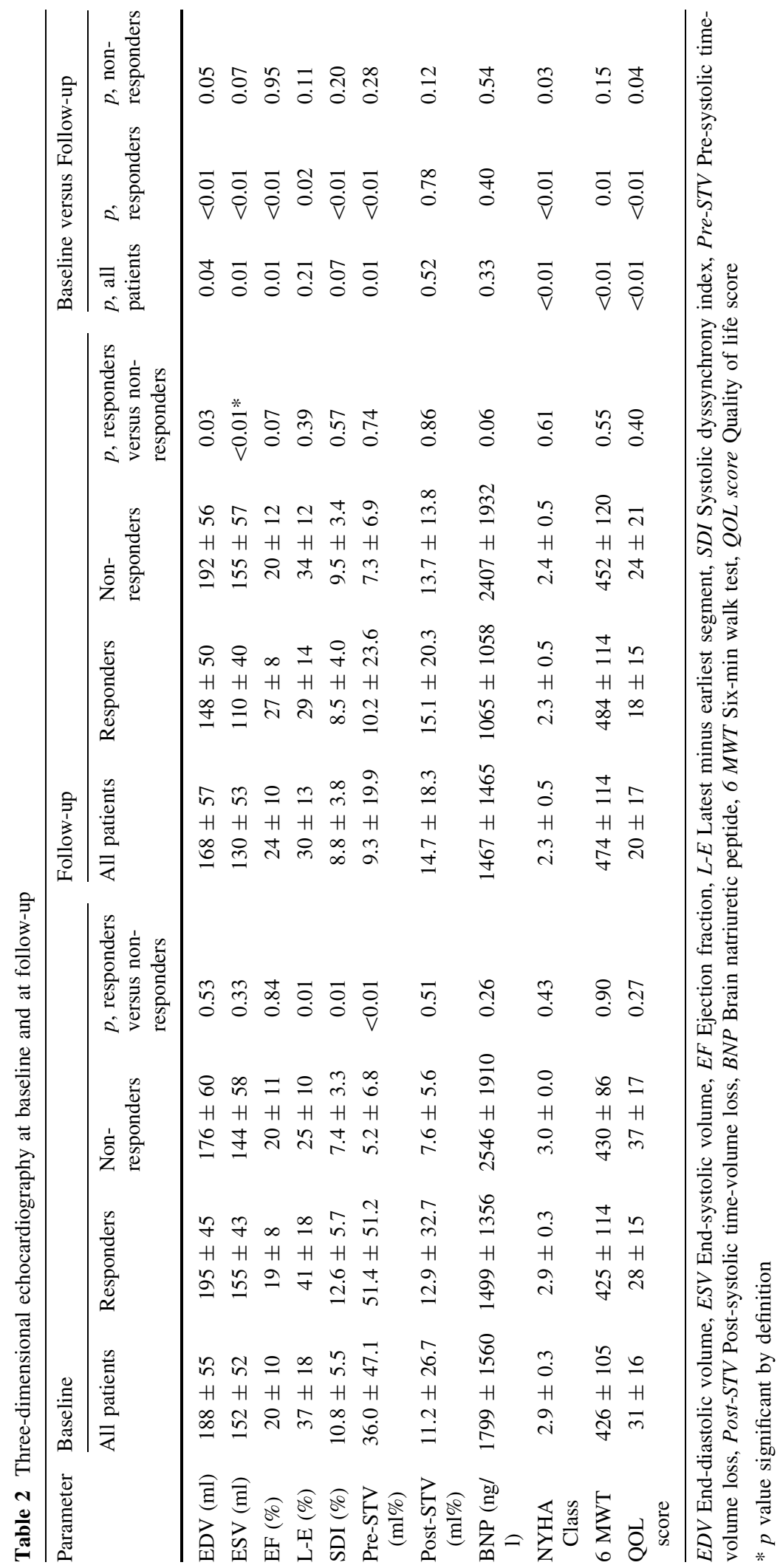


Table 3 Predictive values of response to CRT

\begin{tabular}{|c|c|c|c|c|c|c|c|c|}
\hline \multirow[t]{2}{*}{ Parameter } & \multirow[t]{2}{*}{$\begin{array}{l}\text { Cut-off } \\
\text { value }(\%)\end{array}$} & \multirow[t]{2}{*}{$\begin{array}{l}\text { Sensitivity } \\
(\%)\end{array}$} & \multirow[t]{2}{*}{$\begin{array}{l}\text { Specificity } \\
(\%)\end{array}$} & \multirow[t]{2}{*}{$\begin{array}{l}\text { NPV } \\
(\%)\end{array}$} & \multirow[t]{2}{*}{$\begin{array}{l}\text { PPV } \\
(\%)\end{array}$} & \multirow[t]{2}{*}{$\begin{array}{l}\text { Area under ROC } \\
\text { curve }(95 \% \mathrm{CI})\end{array}$} & \multicolumn{2}{|c|}{$\begin{array}{l}\text { Correlation } \\
\text { to } \Delta \text {-ESV }\end{array}$} \\
\hline & & & & & & & $r$ & $p$ \\
\hline L-E & 24 & 83 & 56 & 78 & 89 & $0.76(0.58-0.94)$ & -0.43 & 0.02 \\
\hline SDI & 8.2 & 67 & 78 & 54 & 86 & $0.81(0.64-0.98)$ & -0.40 & 0.04 \\
\hline Pre-STV & $9.1 \mathrm{ml}$ & 94 & 78 & 88 & 89 & $0.93(0.82-1.00)$ & -0.55 & $<0.01$ \\
\hline Post-STV & $15.3 \mathrm{ml}$ & 22 & 89 & 36 & 80 & $0.37(0.14-0.60)$ & -0.30 & 0.14 \\
\hline
\end{tabular}

L-E Latest minus earliest segment, SDI Systolic dyssynchrony index, Pre-STV Pre-systolic time-volume loss, Post-STV Post-systolic time-volume loss, $N P V$ Negative Predictive Value, $P P V$ Positive Predictive Value, $\Delta-E S V$ end-systolic volume change at follow-up

correlation to reverse remodeling, defined as ESV change at follow-up, with the highest correlation for Pre-STV. Area under ROC curve of Post-STV was not significantly higher than 0.5 . There was no correlation between Post-STV and reverse remodeling.

Bland-Altman plots of reproducibility of SDI, L-E and Pre-STV are shown in Fig. 2. On close examination of the Pre-STV plots, it appears that low Pre-STV measurements are very reproducible. However, we did not perform statistical analysis on this specific issue due to the low number of patients.

\section{Discussion}

The present study is the first to demonstrate the capacity of 3DE to assess mechanical dyssynchrony combined with segmental contractility to predict response to CRT in heart failure patients.

While CRT is a well-established therapeutic approach in moderated-to-severe heart failure patients with reduced LVEF $(\leq 35 \%)$ and wide QRS ( $\geq 120 \mathrm{~ms}$ ) [1], one-third of patients fail to improve clinically or according to remodelling parameters such as ESV after the implantation of a CRT device. The QRS duration only assesses electrical dyssynchrony and it is use for a selection of patients eligible for CRT has been questioned [18-20].

Several studies focused on echocardiographic indices of mechanical dyssynchrony in heart failure patients to predict response to CRT, defined as a reduction in ESV at follow-up. In single-center studies, reasonable predictive values were reported for very distinct echocardiographic parameters: time difference between 4 basal segments, and standard deviations of time with TDI (in a 12-segment model) and 3DE (in 16- or 17-segment model) [10-12, 15, 2127]. However, different echocardiographic techniques for assessment of cardiac mechanical dyssynchrony are not interchangeable and conflicting reports exist on their correlations $[9,15,16,28]$. In the PROSPECT study [13], the only measure that demonstrated predictive value was time difference between lateral and septal peak systolic wall velocity as measured by TDI; it achieved an area under ROC curve of only 0.61 $(p=0.01)$. However, new approaches like the one we tested might help in finding a simple, robust parameter to add to the current criteria proposed by guidelines to decide upon or not to propose to a patient a CRT device.

The present study investigates cardiac mechanical dyssynchrony fundamentally differently from all previous studies, and is proof-of-concept of assessment of mechanical dyssynchrony using parameters that are not only dependent of segmental timing, but also on (decreased) segmental endocardial inward motion. It is conceivable that non-responders with high SDIs might have dyssynchrony mainly in hypokinetic segments. By correcting SDI for regional contractility, STV can provide more comprehensive information on the severity of mechanical dyssynchrony with the ultimate goal of improving the prediction of response to CRT.

\section{STV and SDI}

Correlation between STV and SDI is moderate because STV and SDI are fundamentally different parameters. Firstly, STV is calculated by summation of values of all segments, whereas SDI is a standard deviation $[15,16,26]$. The use of a standard deviation requires a large number of normally distributed data, 
Fig. 2 Reproducibility of LV dyssynchrony parameters. Intra-observer variability Inter-observer variability. On the horizontal axis, average value of 2 observations are shown, and on the vertical axis, difference between observations. The dashed bars represent $95 \%$ confidence intervals. $L-E$ Latest minus earliest segment, SDI Systolic dyssynchrony index, Pre-STV Pre-systolic time-volume loss
Intra-observer variability
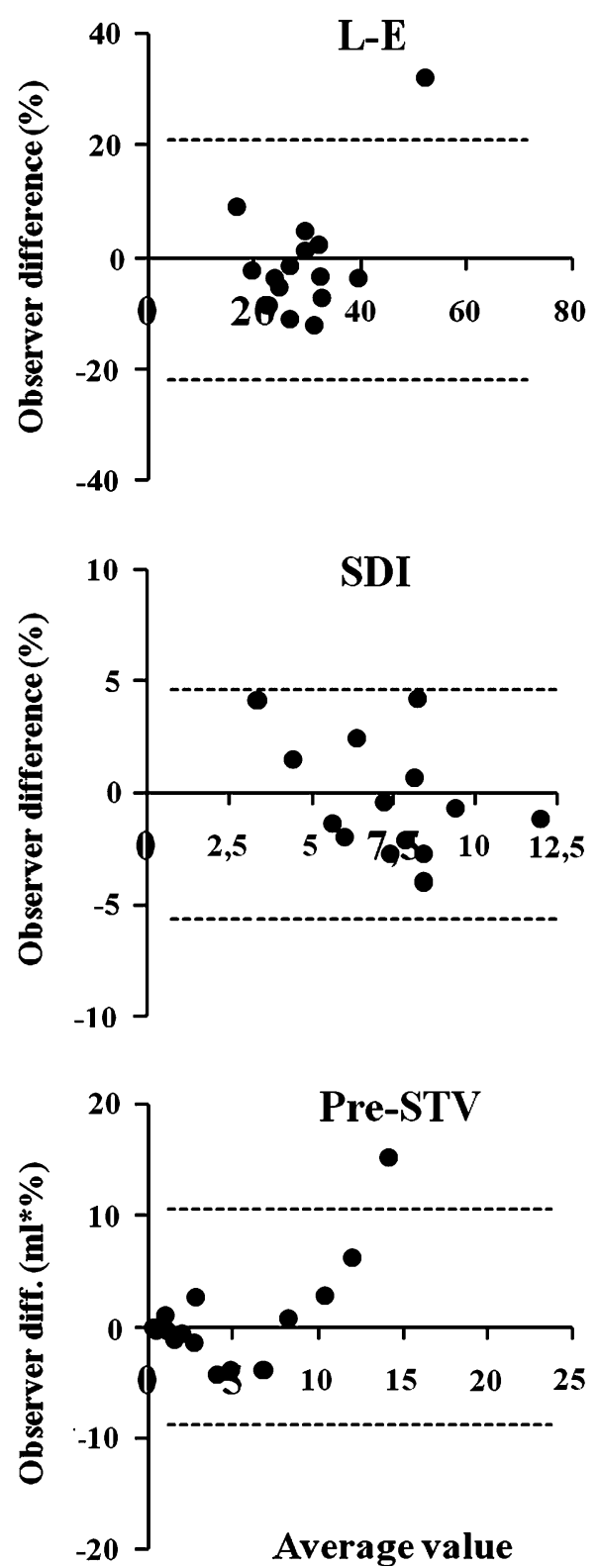
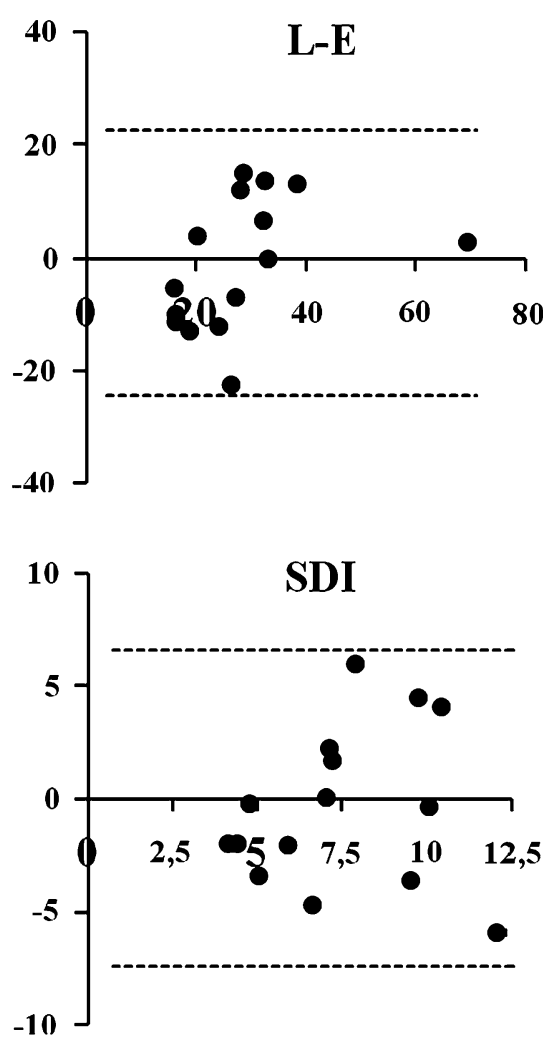

Inter-observer variability

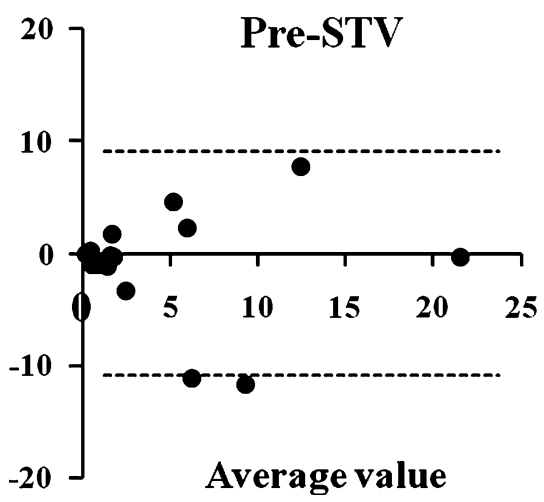

but in SDI there are only 12-17 segments. Presence of a small number of segments with a long delay might cause skewed distribution, impairing robustness of a standard deviation, which is a weakness intrinsic to all standard-deviation based models of mechanical dyssynchrony.

Secondly, since basal segments have higher volumes than the mid- and apical segments, their contribution to the total value of STV is greater than that of mid- and apical segments. This is in contrast to methods that use only timing parameters and where basal, mid- and apical segments contribute equally to the final value.

Finally, reproducibility of SDI is moderate in both the present and in previous studies [7, 8, 15], and as a consequence, SDI values in non-responders and responders vary widely between studies, with partial overlap. SDI in non-responders varies from 3.4 to 
$7.1 \%$, SDI in responders varies from 9.7 to $16.6 \%$ [15, 25-27] and cut-off values between responders and non-responders vary from 5.6 to $10 \%$. It must be emphasised that calculation of all three parameters (L-E, SDI and Pre-STV) was based on the same endocardial borders; reproducibility of SDI and PreSTV seems to be of a similar magnitude. Close observation of Fig. 2 however, reveals that reproducibility of Pre-STV was very high when values were low (the cluster of dots around the 0.0 mark), which implies that absence of any significant amount of PreSTV can be measured very reliably.

\section{Left ventricular dyssynchrony}

In agreement with our results regarding the changes observed in Pre-STV, similar changes were found in a TDI study on heart failure patients received CRT, where peak systolic contraction in 6 basal segments was delayed homogeneously to a timing close to that of the latest segment, so that regional variation after CRT was abolished [5]. This seems to contradict the theory that CRT improves LV function by activating late segments earlier. However, this observation may be explained if we consider that the timing of pacing occurs at end-diastole, and dyssynchrony is assessed at end-systole. We were unable to determine whether the decrease in Pre-STV was caused by a leftward shift of the global time-volume curve or by "true" decrease of Pre-STV. In hemodynamic studies on CRT, an increased positive $\mathrm{dP} / \mathrm{dT}$ was reported [29-31], which might be consistent with the decrease in Pre-STV that we observed. Because events before global endsystole occur during high intraventricular pressure and events after global end-systole occur during low intraventricular pressure, a decrease in Pre-STV might have a larger beneficial influence on myocardial energy efficiency than a decrease in Post-STV.

\section{Future perspectives}

In prediction of response to CRT, the key issue remains accurate and reproducible assessment of left ventricular mechanical dyssynchrony in both contractility and in timing. With minor adaptations in analysis software, (Pre)-STV may be visualised in a polar map, which may aid in optimising biventricular pacemaker lead location. In future studies, time difference between basal, mid- and apical segments [10] and time difference between longitudinal, radial and circumferential contraction [32] should be compared with (Pre) STV or similar measures in prediction of response to CRT. Although speculative, methods that rely on regions-of-interest in combination with tissue Doppler or speckle tracking might obviate the need for endocardial border detection altogether. The present analysis method might be applicable to other imaging modalities as well. Total scar burden, as assessed using contrast-enhanced magnetic resonance imaging, is an important factor influencing response to CRT and may be included in the selection process for CRT candidates [33]. Strain by 3D speckle tracking might be an alternative for the assessment of myocardial necrosis [34] and is also helpful to guide lead position. Systematic evaluation of mitral valve regurgitation [35] might reveal additional information.

\section{Limitations}

The present study was not a randomized, controlled study, but rather a hypothesis generating investigation of a new and still unproven method in a small patient group with a relatively short follow-up period. At present, this method should not be used clinically without further investigation.

Further limitations were in the nature of the software. End-systole was defined as the moment of minimal LV volume. No attention was given to opening or closing of mitral and aortic valves and the isovolumetric relaxation time was not assessed. Although Post-STV is a measure of early diastolic function, we were not able to investigate the events that occur around end-diastole. We could not evaluate if segments in the vicinity of the (postero)lateral pacemaker lead showed the highest Pre- or Post-STV, if they showed the most improvement, or if this had any influence on response to CRT.

\section{Conclusions}

The present method which integrates segmental timing and wall motion might describe LV mechanical dyssynchrony better than methods based solely on timing. In our patient group, Pre-STV might serve as a correction for regional contractility of SDI, and is a better predictor of volume response to CRT than SDI 
or L-E. Larger scale studies are needed to confirm its prognostic value.

\section{Conflicts of interest None.}

Open Access This article is distributed under the terms of the Creative Commons Attribution License which permits any use, distribution, and reproduction in any medium, provided the original author(s) and the source are credited.

\section{References}

1. Freemantle N, Tharmanathan P, Calvert MJ, Abraham WT, Ghosh J, Cleland JG (2006) Cardiac resynchronization for patients with heart failure due to left ventricular systolic dysfunction-a systematic review and meta-analysis. Eur Heart J 8:433-440

2. Daubert JC, Leclercq C, Donal E, Mabo P (2006) Cardiac resynchronisation therapy in heart failure: current status. Heart Fail Rev 11:147-154

3. Takeuchi M, Jacobs A, Sugeng L, Nishikage T, Nakai H, Weinert L, Salgo IS, Lang RM (2007) Assessment of left ventricular dyssynchrony with real-time 3-dimensional echocardiography: comparison with Doppler tissue imaging. J Am Soc Echocardiogr 20(12):1321-1329

4. Chung ES, Leon AR, Tavazzi L, Sun JP, Nihoyannopoulos P, Merlino $\mathrm{J}$ et al (2008) Results of the predictors of response to CRT (PROSPECT) trial. Circulation 117: 2608-2616

5. Yu CM, Chau E, Sanderson JE, Fan K, Tang MO, Fung WH et al (2002) Tissue Doppler echocardiographic evidence of reverse remodeling and improved synchronicity by simultaneously delaying regional contraction after biventricular pacing therapy in heart failure. Circulation 105(4):438-445

6. Bax JJ, Marwick TH, Molhoek SG, Bleeker GB, van Erven L, Boersma E, Steendijk P, van der Wall EE, Schalij MJ (2003) Left ventricular dyssynchrony predicts benefit of cardiac resynchronisation therapy in patients with end-stage heart failure before pacemaker implantation. Am J Cardiol 92(10): 1238-1240

7. Delgado V, Sitges M, Vidal B, Silva E, Azqueta M, Tolosana JM, Mont L, Paré C, Brugada J (2008) Assessment of left ventricular dyssynchrony by real-time three-dimensional echocardiography. Rev Esp Cardiol 61(8):825-834

8. van Dijk J, Dijkmans PA, Götte MJ, Spreeuwenberg MD, Visser CA, Kamp O (2008) Evaluation of global left ventricular function and mechanical dyssynchrony in patients with an asymptomatic left bundle branch block: a real-time 3D echocardiography study. Eur J Echocardiogr 9(1):40-46

9. Burgess MI, Jenkins C, Chan J, Marwick TH (2007) Measurement of left ventricular dyssynchrony in patients with ischaemic cardiomyopathy: a comparison of real-time three-dimensional and tissue Doppler echocardiography. Heart 93(10):1191-1196

10. Bax JJ, Bleeker GB, Marwick TH, Molhoek SG, Boersma E, Steendijk P, van der Wall EE, Schalij MJ (2004) Left ventricular dyssynchrony predicts response and prognosis after cardiac resynchronisation therapy. J Am Coll Cardiol 44(9):1834-1840

11. Kleijn SA, van Dijk J, de Cock CC, Allaart CP, van Rossum AC, Kamp O (2009) Assessment of intraventricular mechanical dyssynchrony and prediction of response to cardiac resynchronization therapy: comparison between tissue Doppler imaging and real-time three-dimensional echocardiography. J Am Soc Echocardiogr 22(9):10471054

12. Yu CM, Fung JW, Zhang Q, Chan CK, Chan YS, Lin H et al (2004) Tissue Doppler imaging is superior to strain rate imaging and postsystolic shortening on the prediction of reverse remodeling in both ischemic and nonischemic heart failure after cardiac resynchronisation therapy. Circulation 110(1):66-73

13. Chung ES, Leon AR, Tavazzi L, Sun JP, Nihoyannopoulos P, Merlino J, Abraham WT, Ghio S, Leclercq C, Bax JJ, Yu CM, Gorcsan J III, St John Sutton M, De Sutter J, Murillo J (2008) Results of the predictors of response to CRT (PROSPECT) trial. Circulation 117(20):2608-2616

14. van der Heide JA, Mannaerts HF, Spruijt HJ, van Campen LM, de Cock C, Visser CA et al (2004) Noninvasive mapping of left ventricular electromechanical asynchrony by three-dimensional echocardiography and semi-automatic contour detection. Am J Cardiol 94(11):1449-1453

15. Kapetanakis S, Kearney MT, Siva A, Gall N, Cooklin M, Monaghan MJ (2005) Real-time three-dimensional echocardiography: a novel technique to quantify global left ventricular mechanical dyssynchrony. Circulation 112(7): 992-1000

16. Zhang Q, Yu CM, Fung JW, Zhang Y, Chan YS, Chan HC et al (2005) Assessment of the effect of cardiac resynchronisation therapy on intraventricular mechanical synchronicity by regional volumetric changes. Am J Cardiol 95(1): 126-129

17. Yu CM, Bleeker GB, Fung JW, Schalij MJ, Zhang Q, van der Wall EE et al (2005) Left ventricular reverse remodeling but not clinical improvement predicts long-term survival after cardiac resynchronisation therapy. Circulation 112(11): 1580-1586

18. Ghio S, Constantin C, Klersy C, Serio A, Fontana A, Campana C, Tavazzi L (2004) Interventricular and intraventricular dyssynchrony are common in heart failure patients, regardless of QRS duration. Eur Heart J 25:571578

19. Donal E, Tournoux F, Leclercq C, De Place C, Solnon A, Derumeaux G et al (2008) Assessment of longitudinal and radial ventricular dyssynchrony in ischemic and nonischemic chronic systolic heart failure: a two-dimensional echocardiographic speckle-tracking strain study. J Am Soc Echocardiogr 21:58-65

20. Bernard A, Donal E, Leclercq C, Ollivier R, Schnell F, de Place $C$ et al. (2009) Impact of right ventricular contractility on left ventricular dyssynchrony in patients with chronic systolic heart failure. Int J Cardiol (in press)

21. Pitzalis MV, Iacoviello M, Romito R, Massari F, Rizzon B, Luzzi G, Guida P, Andriani A, Mastropasqua F, Rizzon P (2002) Cardiac resynchronisation therapy tailored by echocardiographic evaluation of ventricular asynchrony. J Am Coll Cardiol 40(9):1615-1622 
22. Yu CM, Zhang Q, Chan YS, Chan CK, Yip GW, Kum LC et al (2006) Tissue Doppler velocity is superior to displacement and strain mapping in predicting left ventricular reverse remodelling response after cardiac resynchronisation therapy. Heart 92(10): 1452-1456

23. Yu CM, Zhang Q, Fung JW, Chan HC, Chan YS, Yip GW, Kong SL, Lin H, Zhang Y, Sanderson JE (2005) A novel tool to assess systolic asynchrony and identify responders of cardiac resynchronisation therapy by tissue synchronization imaging. J Am Coll Cardiol 45(5):677-684

24. Porciani MC, Lilli A, Macioce R, Cappelli F, Demarchi G, Pappone A, Ricciardi G, Padeletti L (2006) Utility of a new left ventricular asynchrony index as a predictor of reverse remodelling after cardiac resynchronisation therapy. Eur Heart J 27(15):1818-1823

25. Marsan NA, Bleeker GB, Ypenburg C, Van Bommel RJ, Ghio S, Van de Veire NR, Delgado V, Holman ER, van der Wall EE, Schalij MJ, Bax JJ (2008) Real-time threedimensional echocardiography as a novel approach to assess left ventricular and left atrium reverse remodeling and to predict response to cardiac resynchronisation therapy. Heart Rhythm 5(9):1257-1264

26. Marsan NA, Bleeker GB, Ypenburg C, Ghio S, van de Veire NR, Holman ER, van der Wall EE, Tavazzi L, Schalij MJ, Bax JJ (2008) Real-time three-dimensional echocardiography permits quantification of left ventricular mechanical dyssynchrony and predicts acute response to cardiac resynchronisation therapy. J Cardiovasc Electrophysiol 19(4):392-399

27. Soliman OI, Geleijnse ML, Theuns DA, van Dalen BM, Vletter WB, Jordaens LJ, Metawei AK, Al-Amin AM, ten Cate FJ (2009) Usefulness of left ventricular systolic dyssynchrony by real-time three-dimensional echocardiography to predict long-term response to cardiac resynchronisation therapy. Am J Cardiol 103(11):1586-1591

28. Park SM, Kim KC, Jeon MJ et al (2007) Assessment of left ventricular asynchrony using volume-time curves of 16 segments by real-time 3 dimensional echocardiography: comparison with tissue Doppler imaging. Eur J Heart Fail 9(1):62-67

29. Nelson GS, Berger RD, Fetics BJ et al (2000) Left ventricular or biventricular pacing improves cardiac function at diminished energy cost in patients with dilated cardiomyopathy and left bundle-branch block. Circulation 102(25):3053-3059

30. Hay I, Melenovsky V, Fetics BJ et al (2004) Short-term effects of right-left heart sequential cardiac resynchronisation in patients with heart failure, chronic atrial fibrillation, and atrioventricular nodal block. Circulation 110(22):3404-3410

31. van Dijk J, Knaapen P, Russel IK, Hendriks T, Allaart CP, de Cock CC, Kamp O (2008) Mechanical dyssynchrony by $3 \mathrm{D}$ echo correlates with acute haemodynamic response to biventricular pacing in heart failure patients. Europace 10(1):63-68

32. Gorcsan J III, Tanabe M, Bleeker GB, Suffoletto MS, Thomas NC, Saba S, Tops LF, Schalij MJ, Bax JJ (2007) Combined longitudinal and radial dyssynchrony predicts ventricular response after resynchronisation therapy. J Am Coll Cardiol 50(15):1476-1483

33. Ypenburg C, Roes SD, Bleeker GB, Kaandorp TA, de Roos A, Schalij MJ, van der Wall EE, Bax JJ (2007) Effect of total scar burden on contrast-enhanced magnetic resonance imaging on response to cardiac resynchronisation therapy. Am J Cardiol 99(5):657-660

34. Hayat D, Kloeckner M, Nahum J, Ecochard-Dugelay E, Dubois-Randé JL, Jean-François D, Guéret P, Lim P (2011) Comparison of real-time three-dimensional speckle tracking to magnetic resonance imaging in patients with coronary heart disease. Am J Cardiol 109(2):180-186

35. Naqvi TZ, Rafique AM, Swerdlow C, Verma S, Siegel RJ, Tolstrup K, Kerwin W, Goodman J, Gallik D, Gang E, Peter CT (2008) Predictors of reduction in mitral regurgitation in patients undergoing cardiac resynchronisation treatment. Heart 94(12):1580-1588 